\title{
Decomposition of halogenated nucleobases by surface plasmon resonance excitation of gold nanoparticles ${ }^{\star, \star \star}$
}

\author{
Telma S. Marques ${ }^{1,2, a}$, Małgorzata A. Śmiałek ${ }^{2,3, b}$, Robin Schürmann ${ }^{4}$, Ilko Bald ${ }^{4}$, Maria Raposo $^{1}$, Sam Eden $^{2}$, and \\ Nigel J. Mason ${ }^{2,5}$ \\ ${ }^{1}$ CEFITEC, Departamento de Física, Faculdade de Ciências e Tecnologia, Universidade Nova de Lisboa, 2829-516 Caparica, \\ Portugal \\ 2 School of Physical Sciences, The Open University, Walton Hall, MK7 6AA Milton Keynes, UK \\ 3 Department of Control and Power Engineering, Faculty of Ocean Engineering and Ship Technology, Gdansk University of \\ Technology, Gabriela Narutowicza 11/12, 80-233 Gdansk, Poland \\ 4 Physical Chemistry, Institute of Chemistry, University of Potsdam, Karl-Liebknecht-Str. 24-25, 14476 Potsdam-Golm, \\ Germany \\ 5 School of Physical Sciences, University of Kent at Canterbury, Kent CT2 7NH, UK
}

Received 10 April 2020 / Received in final form 3 August 2020 / Accepted 9 September 2020 Published online 1 November 2020

(c) The Author(s) 2020. This article is published with open access at Springerlink.com

\begin{abstract}
Halogenated uracil derivatives are of great interest in modern cancer therapy, either as chemotherapeutics or radiosensitisers depending on their halogen atom. This work applies UV-Vis spectroscopy to study the radiation damage of uracil, 5-bromouracil and 5-fluorouracil dissolved in water in the presence of gold nanoparticles upon irradiation with an Nd:YAG ns-pulsed laser operating at $532 \mathrm{~nm}$ at different fluences. Gold nanoparticles absorb light efficiently by their surface plasmon resonance and can significantly damage DNA in their vicinity by an increase of temperature and the generation of reactive secondary species, notably radical fragments and low energy electrons. A recent study using the same experimental approach characterized the efficient laser-induced decomposition of the pyrimidine ring structure of 5-bromouracil mediated by the surface plasmon resonance of gold nanoparticles. The present results show that the presence of irradiated gold nanoparticles decomposes the ring structure of uracil and its halogenated derivatives with similar efficiency. In addition to the fragmentation of the pyrimidine ring, for 5-bromouracil the cleavage of the carbon-halogen bond could be observed, whereas for 5-fluorouracil this reaction channel was inhibited. Locally-released halogen atoms can react with molecular groups within DNA, hence this result indicates a specific mechanism by which doping with 5-bromouracil can enhance DNA damage in the proximity of laser irradiated gold nanoparticles.
\end{abstract}

\section{Introduction}

For several decades, a substantial research effort has been devoted to finding the most efficient and effective radiosensitisers to be used on various types of cancer $[1,2]$. Among others, DNA sensitising using halogenated nucleobases, like 5-bromouracil (5BrU) and 5-fluorouracil (5FU) has proven to be a very promising and versatile approach [3-6]. Recent studies revealed that the former is capa-

* Contribution to the Topical Issue "Atomic Cluster Collisions (2019)", edited by Alexey Verkhovtsev, Pablo de Vera, Nigel J. Mason, Andrey V. Solov'yov.

** Supplementary material in the form of one pdf file available from the Journal web page at https://doi .org/10.1140/epjd/e2020-10208-3

a e-mail: telma.marques@open.ac.uk

b e-mail: smialek@pg.edu.pl ble of replacing thymine in DNA without substantially altering its biological activity [7] while acting as a radiosensitiser enhancing DNA damage both through direct interaction with primary high energy radiation [8] and indirectly, through interaction with the secondary low energy electrons [9-11]. The latter is commonly used as a drug in conventional treatment of solid cancers of breast, skin, colon, stomach and head [4].

The use of AuNPs in cancer therapy also has been a subject of numerous investigations $[1,2,12-17]$ and their sensitising effects on cancer cells, exposed to various types of radiation, after entering cancer cells has already been proven $[18,19]$. In addition, AuNPs exposed to visible laser radiation are being successfully used in dermatology, in nonablative phototherapy of acne vulgaris [20]. Hence it was only a matter of time before the potential of NPs in photothermal cancer therapy (PTT) aided by pulsed laser radiation was realised with $532 \mathrm{~nm}$ light 
a)<smiles>O=c1cc[nH]c(=O)[nH]1</smiles>

b)<smiles>O=c1[nH]cc(F)c(=O)[nH]1</smiles>

c)<smiles>O=c1[nH]cc(Br)c(=O)[nH]1</smiles>

Fig. 1. Chemical structures of the nucleobases used in this work, (a) U, (b) $5 \mathrm{FU}$ and (c) $5 \mathrm{BrU}$.

and demonstrated for use in breast cancer therapy [18,19]. Furthermore, AuNPs have been shown to be photothermally efficient with this type of radiation [22]. For example, Nam et al. [23] designed spiky gold nanoparticles, improving the photothermal efficiency in vitro and in vivo and Dykman et al. [24] presented studies of different coatings of AuNPs to improve their optical proprieties for PTT. Therefore, we decided to test a possible improvement to this promising method by the inclusion of classic radiosensitiser molecules (see Fig. 1) adsorbed on the surface of AuNPs, especially since $5 \mathrm{FU}$ has already been shown to improve the effect of AuNP-enhanced photothermal therapy [25]. AuNPs of $40 \mathrm{~nm}$ in diameter were used in order to test their sensitising capabilities as heatgenerating centres. AuNPs also show a characteristic surface plasmon resonance (SPR) around $530 \mathrm{~nm}$, depending on the size and shape of the particles [26] and the laser wavelength was chosen to match this SPR band associated with AuNPs $[12,27]$.

The aim of this work was, therefore, to gain new understanding into how two effects already known from the literature - radiation sensitization using halogenated nucleobases and using AuNPs - might be combined to create a system that enables more accurate and lethal damage to be delivered to specific parts of cancerous cells.

\section{Experimental methods}

\subsection{Chemicals and solutions}

All the nucleobases (NBs) used: uracil (U; CAS 66-22-8), 5-bromouracil (5BrU; CAS 51-20-7) and 5-fluorouracil (5FU; CAS 51-21-8) were purchased from Sigma-Aldrich, UK. Gold nanoparticles of $40 \pm 3 \mathrm{~nm}$ diameter (AuNPs; CAS EM.GC40) were purchased from British Biocell International (BBI Solutions), UK. The AuNPs were supplied as an aqueous suspension with a concentration of $9.00 \times 10^{10}$ gold particles $/ \mathrm{mL}$. All chemicals were used without further purification. Aqueous solutions and dilutions were prepared with ultra-high purity water (UHPW) with resistivity of $18.2 \mathrm{M} \Omega \mathrm{cm}$, supplied by a Suez-Purite Neptune purification system. The NBs were used at a final concentration of $25 \mu \mathrm{M}$ and the AuNP solution with final concentration of $44.7 \mathrm{pM}$. Samples were prepared freshly before each set of irradiation. In each experiment, $2 \mathrm{~mL}$ of solution was placed in a QS (quartz glass high performance) cuvette (Hellma ${ }^{\circledR}$ Analytics), with $10 \mathrm{~mm} \pm 0.01 \mathrm{~mm}$ of optical path length.

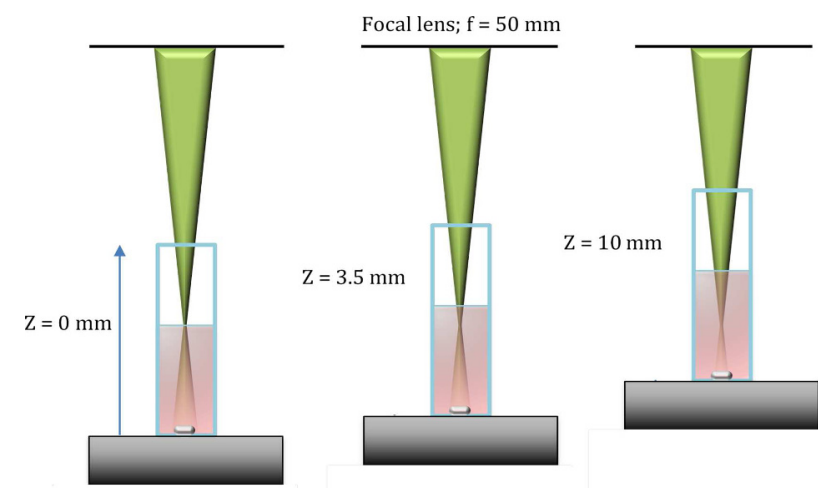

Fig. 2. Illustration of the focal distance $Z$ variation inside the sample irradiated.

\subsection{Irradiation setup}

The experiments were performed within the Molecular Cluster Laboratory in The Open University, United Kingdom. The setup is similar to the one used by Schürmann and co-workers described previously in detail [28] so only the most salient features will be described here.

Briefly, the second harmonic $(532 \mathrm{~nm})$ of a Minilite nanosecond pulsed Nd:YAG laser from Continuum ${ }^{\circledR}$, was chosen as the irradiating light source. In this study, the pulse repetition rate was maintained at $15 \mathrm{~Hz}$ and energy tuned to $16 \mathrm{~mJ}$ per pulse, giving a power of $240 \mathrm{~mW}$. The distance from the focus of the laser beam $(Z)$ can be varied through translation of the platform upon which the sample is placed with movement on the $Z$ axis (3D) from $0 \mathrm{~mm}$, where the beam is focused at the surface of the sample, to $10 \mathrm{~mm}$, roughly halfway through the sample volume (Fig. 2). The average laser fluence at the surface per pulse for each position was calculated through equation (1):

$$
\text { Laser fluence }=\frac{\text { energy per pulse }}{A_{\text {beam }} \times \text { pulse width }}
$$

where the energy per pulse unit is in joules $(\mathrm{J})$, and the pulse width $(\mathrm{FWHM})$ corresponds to $5.0 \times 10^{-9} \mathrm{~s}$, while $A_{\text {beam }}$ is the area of the beam at the surface of the sample. The illuminated volume was obtained from measurement of the $r$ dependence with $Z$ and calculating the resulting revolution solid integral (presented in Supporting Information (SI) SI.1).

The laser beam was focused by the optical system to a surface area of $0.12 \mathrm{~mm}^{2}$ corresponding to the focal distance of $Z$ in $\mathrm{mm}$, where the focus of the beam is at the surface of the sample (Fig. 2). The samples were irradiated from the top whilst being continually stirred to avoid any temperature gradients in the solution Before each set of irradiations, the power of the beam was monitored using a laser power meter (UNO from Gentec-EO).

\subsection{Measurement of photoabsorption spectrum of the solution}

UV-Vis absorbance measurements were performed using a Thermo Scientific $^{\mathrm{TM}}$ Evolution $^{\mathrm{TM}} 201$ (UK) spectrometer 
a)

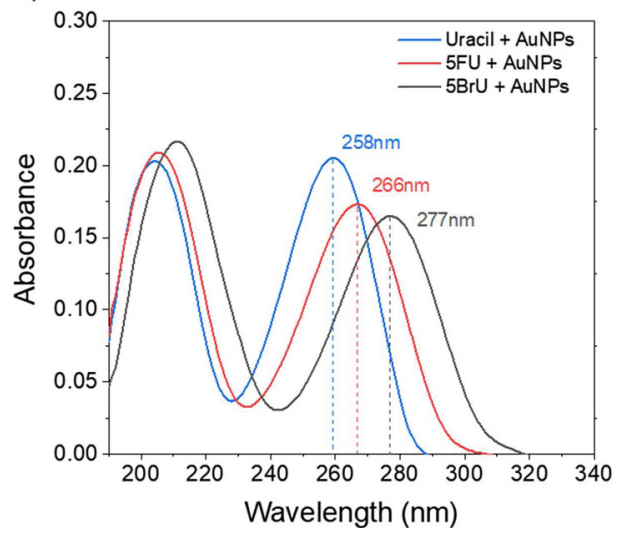

b)

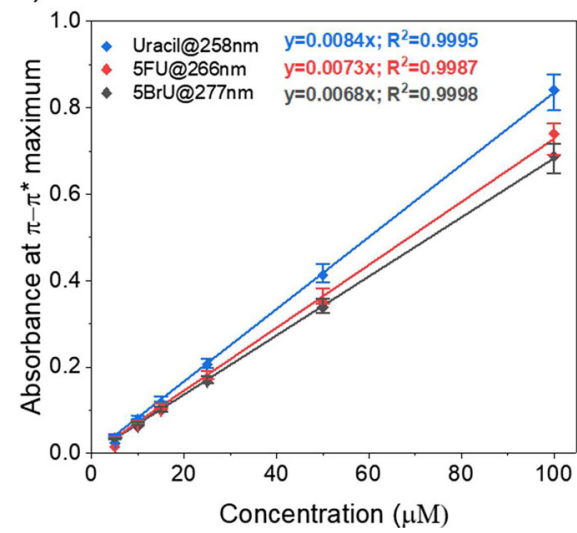

Fig. 3. (a) UV-Vis spectra of aqueous solutions of U, $5 \mathrm{FU}$ and $5 \mathrm{BrU}$ with AuNPs at the concentration of $25 \mu \mathrm{M}$, corrected for AuNPs signal, and (b) standard curves for the NBs in study where the maxima absorbance of the characteristic peaks (258, 266 and $277 \mathrm{~nm}$, respectively) are plotted vs concentration of aqueous solutions NBs+AuNPs.

operating over the range of $190-800 \mathrm{~nm}$, with a bandwidth of $1 \mathrm{~nm}$ and data intervals of $1 \mathrm{~nm}$. The spectrophotometer was placed next to the irradiation apparatus to minimize the time elapsed between irradiation and spectrum acquisition. Therefore, all spectra were acquired after a specific irradiation time and the cuvette placed back on the setup for the next irradiation. All irradiations were performed at room temperature (RT), in triplicate, and equivalent irradiations UHPW samples, in the presence and absence of AuNPs, and without nucleobases were performed as references. The samples were irradiated until the spectrophotometric $\pi-\pi^{*}$ band of the analyte was close to zero absorbance, indicating complete degradation of the NB.

\section{Results and discussion}

\subsection{Absorption coefficient values determination}

In this experimental work, we determine the concentration decrease in the individual NBs during irradiation. It is known that uracil has a characteristic absorption band centred at $255 \mathrm{~nm}$ [29], $5 \mathrm{BrU}$ at $277 \mathrm{~nm}$ [28] and $5 \mathrm{FU}$ at $266 \mathrm{~nm}$ [11]. From the results available in the literature $[10,28,30,31]$ and the first plots obtained experimentally, we observed that there is an overlap of $\pi-\pi^{*}$ signal of NBs with the interband (IB) states of AuNPs. Hence, the determination of the absorption coefficients from the standard curves of the NBs+AuNPs, helps us to infer the probability of degradation due to absorption of light and quantify the concentration of the NBs in the mixed solution after irradiation. Figure 3 a presents an example of the UV-Vis spectra, corrected for AuNPs signal, obtained in aqueous solutions of $\mathrm{U}, 5 \mathrm{FU}$ and $5 \mathrm{BrU}$ with a AuNP concentration of $25 \mu \mathrm{M}$ for $Z=1 \mathrm{~mm}$. These spectra show the maximum absorbance, associated with the $\pi-\pi^{*}$ transitions [31] for the NBs used. The uracil peak is slightly different from that found in references $[9,10,29]$, which may be attributed to a solvent
Table 1. Maximum wavelength and extinction coefficients of $\mathrm{U}, 5 \mathrm{FU}$ and $5 \mathrm{BrU}$.

\begin{tabular}{llll}
\hline Nucleobase & $\pi-\pi^{*}$ & \multicolumn{2}{l}{ Extinction coefficient $\left(\mathrm{M}^{-1} \mathrm{~cm}^{-1}\right)$} \\
\cline { 3 - 4 } & $(\mathrm{nm})$ & Literature & This work \\
\hline Uracil & $260 ; 255$ & $7800-8200[9,10,29]$ & $8400 \pm 562$ \\
$5 \mathrm{FU}$ & 266 & $7000[11]$ & $7300 \pm 659$ \\
$5 \mathrm{BrU}$ & 277 & $7010[10,32]$ & $6800 \pm 400$ \\
\hline
\end{tabular}

effect. To determine the absorption coefficients, the maximum values of absorbance that were achieved for each concentration after background subtraction (namely IB and SPR band) were plotted (Fig. 3b). The correction was obtained through the subtraction of the spectra of the AuNPs solution (the control solution) from the data NB+AuNPs. The standard curves were built for the three NBs, and are the result of the Gaussian fit of the corrected absorption spectra for each NB (Fig. 3b). The linear fit suggests a direct proportionality between maximum absorption and concentration in the range studied $(5-100 \mu \mathrm{M})$.

The experimental extinction coefficients of the NBs were calculated using the linear fit equations presented in Figure 3b, and the values are listed in Table 1. Calculations of extinction coefficient $(\varepsilon)$ are based on the BeerLambert law, $A=\varepsilon \cdot l \cdot c$, where $A$ is the absorbance of the solution at a particular wavelength, $l$ is the length of the optical path and $c$ the concentration of the solution. The extinction coefficient is experimentally calculated using the slope of the linear fit of the absorbance vs concentration spectra of a given compound for a given wavelength, that is measuring $A / c$, with $l$ equal to $1 \mathrm{~cm}$ (from the cuvette specifications).

Table 1 also presents the maximum wavelength $\left(\pi-\pi^{*}\right)$ of the characteristic peak in the UV-Vis spectra for the nucleobases studied (Fig. 3a) as well as the calculated values for the extinction coefficient, where the error presented is the standard deviation of the average. Our values are compared with ones found in the literature. 

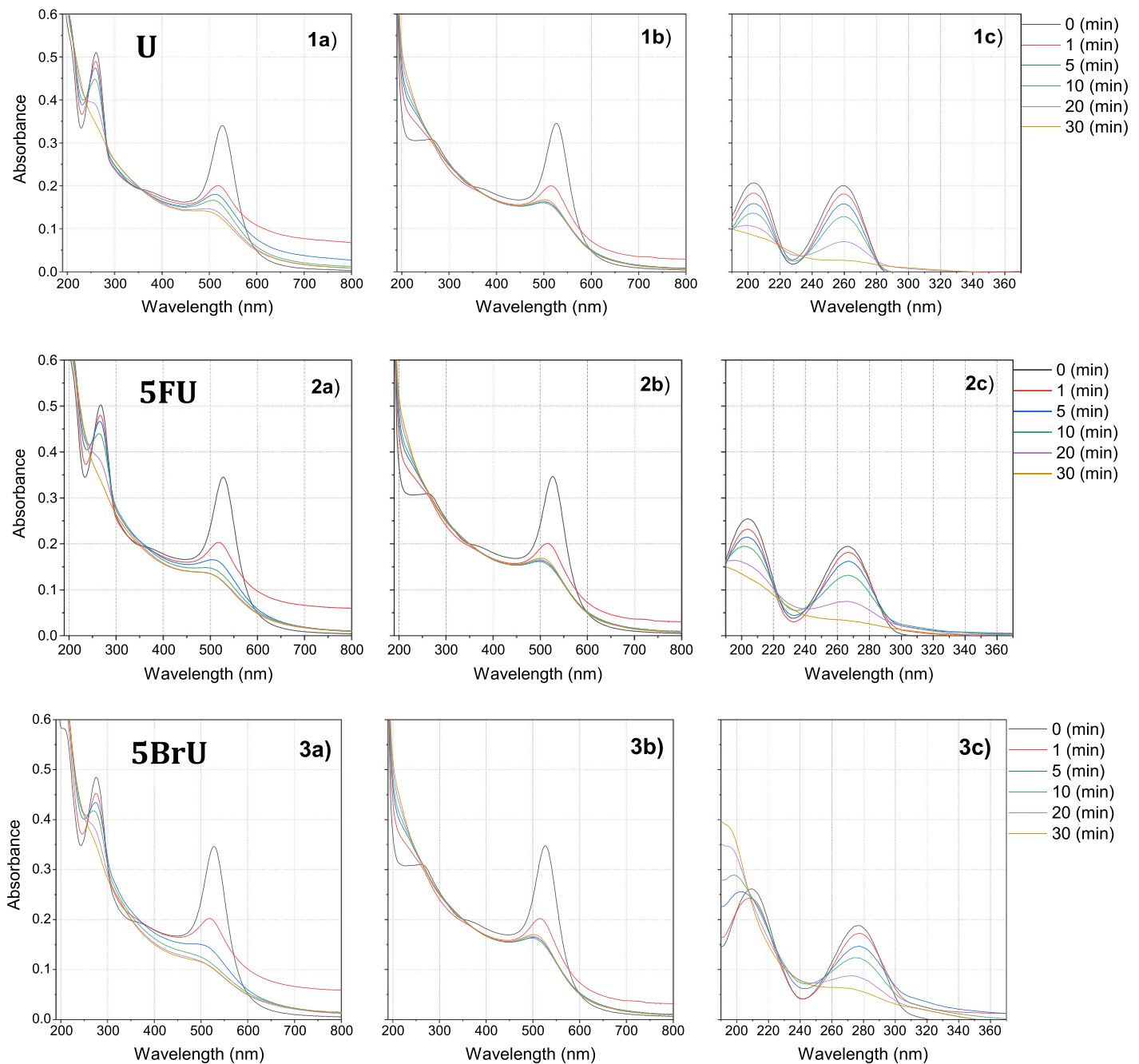

Fig. 4. Absorption spectra of samples following exposure to $532 \mathrm{~nm} \mathrm{Nd}$ :YAG laser radiation for up to $30 \mathrm{~min}$ with $Z=1 \mathrm{~mm}$. The curves from left to right show (a) the raw spectra of solutions containing nucleobases (NB)+AuNPs, (b) the raw spectra of samples containing AuNPs only, and (c) the corrected spectra for U, 5FU, and 5BrU produced by subtracting the AuNP (only spectra b) from the NB+AuNPs spectra (a).

Comparison of the experimental data with the data available in the literature (see Tab. 1) suggests that the present values are equal, considering the uncertainties obtained for each fitted curve. These coefficients suggest that uracil $(\mathrm{U})$ is the molecule that presents the highest capacity to absorb photons, followed by $5 \mathrm{FU}$ and $5 \mathrm{BrU}$. However, the position of the maximum of its absorption band of $U$ is at shorter wavelengths, $258 \mathrm{~nm}$, when compared to the positions of the maximum of the absorption band of the other compounds, 266 and $277 \mathrm{~nm}$ respectively. These results may indicate that the decomposition rate of nucleobases are similar, when the SPR of the AuNPs is excited with the laser light.

\subsection{Photon-induced damage}

The absorbance of $\mathrm{U}, 5 \mathrm{FU}$ and $5 \mathrm{BrU}$ solutions mixed with AuNPs, for various irradiation times, and of samples containing AuNPs recorded under the same conditions, are shown in columns (a) and (b) in Figure 4. The effect of the irradiation time on the nucleobases is presented in Figure 4 column (c). These spectra are obtained by subtracting from the UV-Vis spectra of the NBs with AuNPs, Figure 4 column (a), the spectra of pure AuNPs, Figure 4 column (b), when irradiated under the same conditions $(Z=1 \mathrm{~mm})$. All the irradiations were measured on the same day to avoid contributions/modifications from external factors and the same control was used for all the samples. The spectra of $\mathrm{U}, 5 \mathrm{FU}$ and $5 \mathrm{BrU}$ are shown in rows (1), (2) and (3), respectively, of Figure 4.

According to Pyatenko et al. [33], the reduction of the AuNPs size can be attributed to Coulomb explosion (or spontaneous fission of the NPs). The excitation of the AuNPs near their SPR leads to electron emission from the AuNPs, resulting in an increase of the temperature and pressure in their vicinity. This loss of electrons leads to Coulomb explosion of the AuNPs, a fission process due to the repulsion of the remaining positive charges in the AuNPs. The decrease in the characteristic SPR band with 


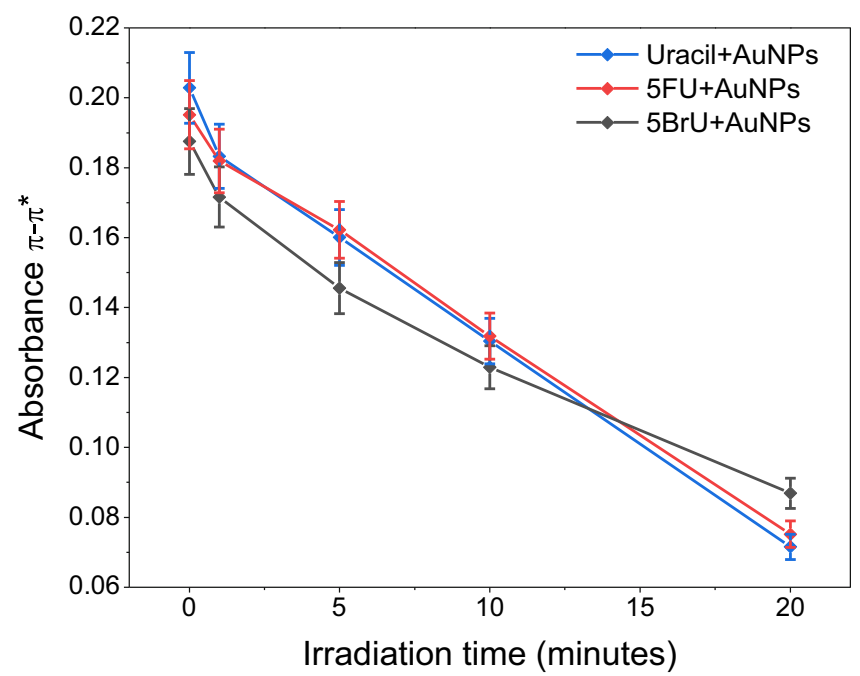

Fig. 5. Maximal absorbance associated with the $\pi-\pi^{*}$ transitions in the corrected spectra of $\mathrm{U}, 5 \mathrm{FU}$ and $5 \mathrm{BrU}$ as a function of the irradiation time with $\mathrm{Nd}$ :YAG at laser fluence $25.5 \times 10^{13} \mathrm{~W} / \mathrm{m}^{2}(Z=1 \mathrm{~mm})$.

increasing laser irradiation time (Fig. 4a) is thus related with the size reduction of the AuNPs, probably due to Coulomb explosions [10].

The corrected spectra of all NBs show that the exposure to the laser light led to the reduction of the absorbance band assigned to $\pi-\pi^{*}$ transitions of these compounds, which is a result of the decomposition of the NBs. The data in column (c) were fitted with a Gaussian curves and the maximum absorbances associated with each $\pi-\pi^{*}$ band are plotted against the irradiation time in Figure 5.

The highest rate of decomposition was observed in uracil, approximately $65 \%$ for the 20 min irradiation time. This is followed by $5 \mathrm{FU}$ with a decrease in the characteristic peak of $61 \%$ and $5 \mathrm{BrU}$ showing a reduction of around $54 \%$. Similar trends were obtained at different sample positions $(Z)$, as shown in Supplementary Material.2.

To analyse the variations occurring in the AuNPs SPR absorption band, the maximum absorption at $532 \mathrm{~nm}$ was plotted for the different sample positions $(Z)$ at the exposure time of $20 \mathrm{~min}$ (Fig. 6). The irradiated volume decreases with sample position due to the focused beam shape. As the sample moves up, the irradiated volume decreases, as shown in Table 2, hence increasing the energy deposited per volume per pulse (i.e. irradiation dose). The dose (and hence the temperature), on the other hand, has an effect on the response of the NPs, namely on the size distribution, which will also dictate the SPR absorption characteristics (area, peak position). A rough estimation of the temperature of the AuNPs during irradiation was made by considering that $1 \%$ of the energy pulse is absorbed by all $40 \mathrm{~nm}$ NPs in the illuminated volume, using bulk Au constants. The size of the AuNPs varies indirectly with the exposure time. The data are not shown here but related information can be found in references González-Rubio et al. and Marques et al. [27,31]. Thus, position $0 \mathrm{~mm}$ will have a larger irradiated volume, a lower dose as well as a lower increase in temperature $(\sim 1700 \mathrm{~K})$ while position 10 has the smallest irradiated volume, a higher dose and a three-fold temperature increase to about $4600 \mathrm{~K}$. The AuNP temperature will affect the dynamics of the destruction (explosion) of the AuNPs and aggregation of the AuNPs. In a separate series of experiments using dynamic light scattering (DLS) and transmission electron microscopy (TEM) measurements (data not shown), it was found that for the irradiation times up to 1 min a normal distribution of NPs centred at $\sim 1 \mathrm{~nm}$ diameter was observed. However, as the dose increases, larger particles of diameter greater than $30 \mathrm{~nm}$ are also observed [30].

The maximum absorbance at the characteristic wavelength for each NB+AuNPs was plotted against laser fluence (Fig. 6a). The data show that the absorbance by $5 \mathrm{BrU}$ post-irradiation shows a relatively weak dependence on laser fluence compared with $\mathrm{U}$ and 5FU. The latter molecules present a very similar trend, and all the NBs have a minimal absorption at $10^{13} \mathrm{~W} / \mathrm{m}^{2}$ (at $Z=$ $3.5 \mathrm{~mm}$ ), fluencies where AuNPs present a low absorption caused by their decreased diameter after extensive photothermal decomposition of initial NPs, resulting in a large decrease in the plasmon band.

As the laser fluences increases (positions 5 and $10 \mathrm{~mm}$ ), the average size of the particles increases due to coalescence, thus increasing the intensity of the SPR absorption band. This may indicate that for higher area to volume ratio (positions 0 to $3.5 \mathrm{~mm}$ ) the reaction occurs at a higher rate. This rate decreases as some large particles are formed (positions 5 and $10 \mathrm{~mm}$ ), which decreases the area/volume ratio. It is worth noting that at positions 5 and $10 \mathrm{~mm}$ the boiling temperature of bulk gold $(2792 \mathrm{~K})$ is exceeded. In general, a clear correlation between the decreased size of the fragmented AuNPs and the decomposition rate of the nucleobases has been observed.

\subsection{Do the AuNPs enhance the laser irradiation effect?}

To answer this question, we have exposed three NBs to laser radiation under the same conditions. As discussed previously, we have chosen the position of $Z=1 \mathrm{~mm}$, and $20 \mathrm{~min}$ as the maximum of exposure period. For each compound we have performed the experiments in the absence of AuNPs, using the same final concentration of NB. The data analysis applied to each spectrum follows the same rationale used for the samples with AuNPs, however here the control is only UHPW irradiated under the same conditions as the samples and is presented in detail in Supplementary Material.3.

Figure 7a was generated from the Gaussian fit to the $\pi-\pi^{*}$ transitions of the NBs varying with time in the absence of AuNPs, and Figure $7 \mathrm{~b}$ is the same data shown in Figure 5, NBs solutions with AuNPs up to 20 min of irradiation.

The results clearly show that the presence of AuNPs during the irradiation with the $532 \mathrm{~nm}$ Nd:YAG laser dramatically enhances the degradation of all the nucleobases under study as compared to nucleobases irradiated under the same conditions in the absence of AuNPs. The percentage variation of the $\pi-\pi^{*}$ transitions for the NBs was 
a)

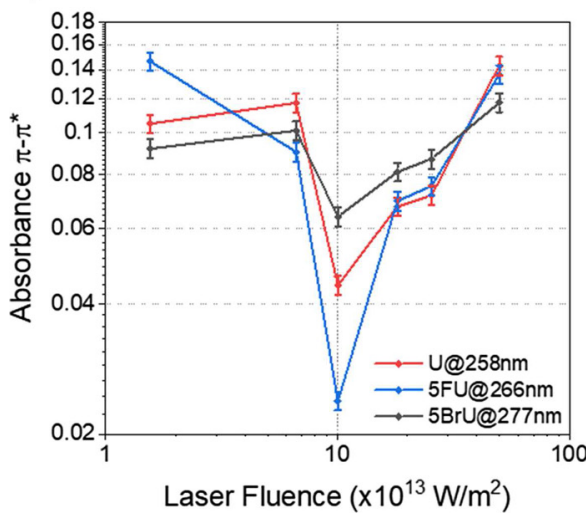

b)

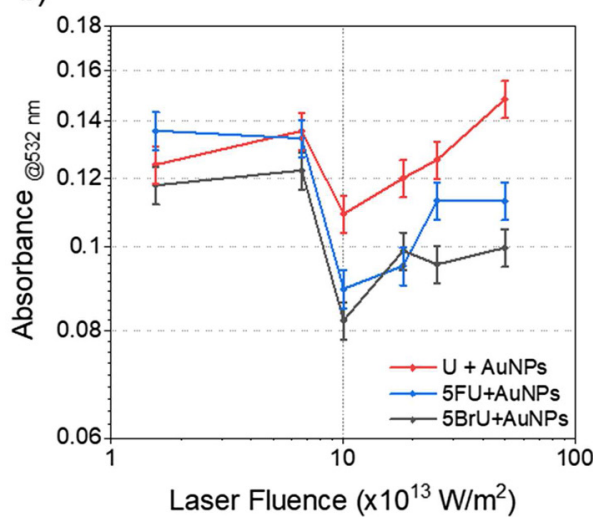

Fig. 6. Maximum absorbance of (a) solutions of uracil (red), $5 \mathrm{FU}$ (blue) and 5BrU (grey) with AuNPs, at 258,266 and $277 \mathrm{~nm}$, respectively, after 20 min of laser illumination, and (b) absorption of AuNPs solutions with uracil (red), 5FU (blue) and 5BrU (grey) for the characteristic SPR at $532 \mathrm{~nm}$, after $20 \mathrm{~min}$ of laser illumination, plotted against the laser fluence.

Table 2. Variation of the irradiated volume and temperature of the AuNPs with sample position.

\begin{tabular}{llllr}
\hline $\begin{array}{l}\text { Position } \\
Z(\mathrm{~mm})\end{array}$ & $\begin{array}{l}\text { Surface fluence } \\
\left(\mathrm{W} / \mathrm{m}^{2}\right)\end{array}$ & $\begin{array}{l}\text { Illuminated } \\
\text { volume }\left(\mathrm{m}^{3}\right)\end{array}$ & $\begin{array}{l}\text { Illuminated } \\
\text { volume }(\%)\end{array}$ & $\begin{array}{c}\Delta T \\
(\mathrm{~K})\end{array}$ \\
\hline 0 & $40.2 \times 10^{13}$ & $4.2 \times 10^{-08}$ & 2.10 & 1700 \\
1 & $25.5 \times 10^{13}$ & $3.7 \times 10^{-08}$ & 1.85 & 1900 \\
2 & $18.2 \times 10^{13}$ & $3.2 \times 10^{-08}$ & 1.63 & 2200 \\
3.5 & $10.0 \times 10^{13}$ & $2.7 \times 10^{-08}$ & 1.34 & 2700 \\
5 & $6.63 \times 10^{13}$ & $2.2 \times 10^{-08}$ & 1.11 & 3200 \\
10 & $1.56 \times 10^{13}$ & $1.6 \times 10^{-08}$ & 0.78 & 4600 \\
\hline
\end{tabular}

a)

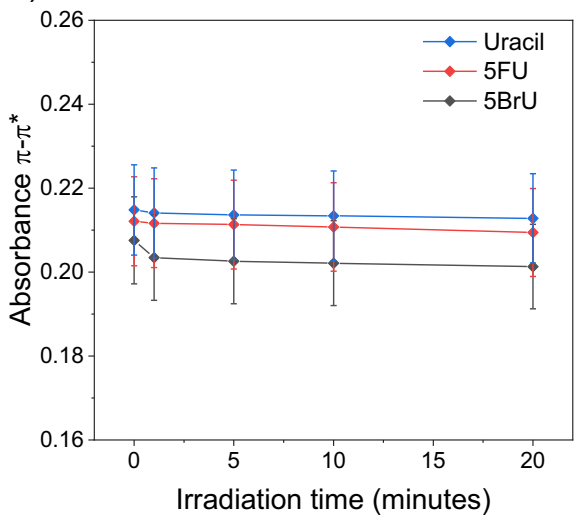

b)

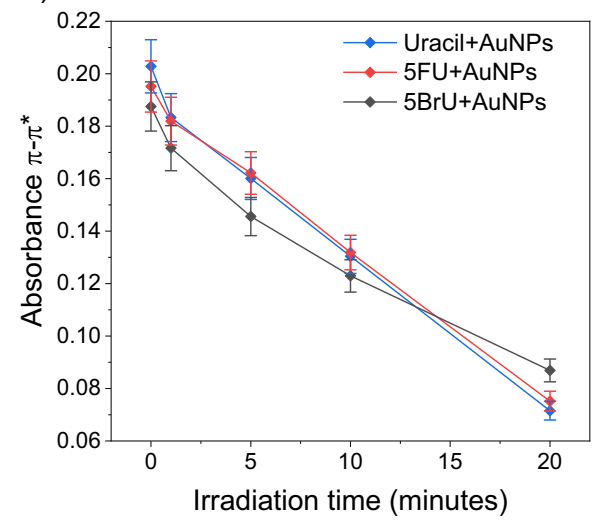

Fig. 7. Changes in the maximum absorbance of the NBs in study after exposure to laser light (a) without AuNPs, and (b) in presence of AuNPs, performed at laser fluence $25.5 \times 10^{13} \mathrm{~W} / \mathrm{m}^{2}(Z=1 \mathrm{~mm})$.

determined through the following equation:

$$
\Delta \mathrm{Abs}=\frac{\mathrm{Abs}_{0}-\mathrm{Abs}_{n}}{\mathrm{Abs}_{0}} \times 100 \%,
$$

where $\Delta \mathrm{Abs}$ is the variation in the absorbance, $\mathrm{Abs}_{0}$ the absorption before the laser illumination and $\mathrm{Abs}_{n}$ corresponds to the absorption measured for a specific irradiation time between 1 and $20 \mathrm{~min}$, the result is presented in Table 3 . In the optical absorption measurements, the Uracil characteristic $\pi-\pi^{*}$ absorption band has the highest absorption coefficient without AuNPs for all irradiation times (Fig. 7a). The coefficients of absorption remain nearly constant for all samples up to the maximum irradiation time. In the absence of AuNPs, the laser light itself does not seem to be able to induce significant decomposition of the NBs. On the other hand, samples with AuNPs showed a continuous decrease in the maximum absorption coefficient with irradiation time (Fig. 7b). This reduction is more pronounced for Uracil. That is, in the presence of AuNPs the NB that absorbs more light 
Table 3. Summary of the reduction (in percentage, \%) of the characteristic band for each nucleobase with the increase of the illumination time $(Z=1 \mathrm{~mm})$.

\begin{tabular}{|c|c|c|c|c|c|c|}
\hline \multirow{2}{*}{$\begin{array}{l}\text { Irradiation } \\
\text { time (min) }\end{array}$} & \multicolumn{3}{|c|}{ Presence of AuNPs } & \multicolumn{3}{|c|}{ Absence of AuNPs } \\
\hline & $\mathrm{U}$ & $5 \mathrm{FU}$ & $5 \mathrm{BrU}$ & $\mathrm{U}$ & $5 \mathrm{FU}$ & $5 \mathrm{BrU}$ \\
\hline 0 & 0 & 0 & 0 & 0 & 0 & 0 \\
\hline 1 & 10 & 7 & 8 & 0 & 2 & 0 \\
\hline 5 & 21 & 17 & 22 & 1 & 2 & 0 \\
\hline 10 & 36 & 32 & 34 & 1 & 3 & 1 \\
\hline 20 & 65 & 61 & 54 & 1 & 3 & 1 \\
\hline
\end{tabular}

also presents the highest reduction of absorption for the maximum time of irradiation.

\subsection{Nucleobases' concentrations}

The results presented in this work show the reduction of the $\pi-\pi^{*}$ absorption band for the three NBs after irradiation in the presence of AuNPs for increasing irradiation time. This demonstrates the decomposition of the nucleobases in the pre-irradiated solution but the experiment is limited in its capacity to identify the specific reaction products. The notable exception is uracil production in the irradiated $5 \mathrm{FU}$ and $5 \mathrm{BrU}$ solutions; an increase in uracil decomposition can be recognized via a modification in the shape of the observed $\pi-\pi^{*}$ bands. We have thus estimated the amount of $5 \mathrm{FU}, 5 \mathrm{BrU}$ and $\mathrm{U}$ that is being formed/decomposed. The Gaussian fit for two peaks with maximum wavelengths of 258 and $266 \mathrm{~nm}$ for each individual spectrum is presented in Figure 4,2c (5FU results), and two peaks with maximum of 258 and $277 \mathrm{~nm}$ for each individual spectrum is presented in Figure 4,3c (5BrU results). The next step was to determine the concentration of each pair $(5 \mathrm{FU}+\mathrm{U}$ and $5 \mathrm{BrU}+\mathrm{U})$ using the linear fit equation employed already in Figure 4,2b, thus creating standard curves for $5 \mathrm{FU}$ and $5 \mathrm{BrU}$. An example of the data analysis for $5 \mathrm{BrU}$ is shown in the plots presented in Figure 8. As the centre of $\pi-\pi^{*}$ band of uracil differs from that of $5 \mathrm{BrU}$ by a greater wavelength than it does from that of $5 \mathrm{FU}$, the concentrations of $5 \mathrm{BrU}+\mathrm{U}$ could be determined with higher precision than that of $5 \mathrm{FU}+\mathrm{U}$.

Figure 9 shows the nucleobase concentrations obtained with increasing irradiation time of $5 \mathrm{FU}$ (plot a) and $5 \mathrm{BrU}$ (plot b) samples with AuNPs. The results demonstrate that while both molecules are strongly decomposed due to the interaction of the laser pulses with the AuNPs, the total amount of $U$ formed during the irradiation is markedly higher from $5 \mathrm{BrU}$ than from $5 \mathrm{FU}$. Furthermore, Figure 9 shows that the total $\pi-\pi^{*}$ band (from $5 \mathrm{FU}$ alone in plot (a) and from $5 \mathrm{BrU}+\mathrm{U}$ in plot (b)) is suppressed more strongly in the $5 \mathrm{FU}$ experiments than in the $5 \mathrm{BrU}$ experiments.

The similar decrease in the concentration of both $5 \mathrm{FU}$ and $5 \mathrm{BrU}$ is attributed to the fragmentation of the pyrimidine ring. This process is nearly linear in Figure 9a and the $5 \mathrm{FU}$ concentration decreases close to zero after 30 min irradiation. The $5 \mathrm{BrU}$ concentration decays with an approximately exponential shape for irradiation times up to 5 min and subsequently shows an approximately linear decay, with a final $(30 \mathrm{~min})$ concentration of about $3 \pm 1 \mu \mathrm{M}$. The trend in the detected uracil levels also differs between the two halogenated uracils. The absence of any discernible $\mathrm{U}$ signal in the $5 \mathrm{FU}$ data set indicates that there is immediate ring fragmentation before any $\mathrm{U}$ can be produced following a dehalogenation process. In the $5 \mathrm{BrU}$ data set, the $\mathrm{U}$ concentration remains close to zero for irradiation times up to $5 \mathrm{~min}$, and then steadily rises during the approximately linear phase of the $5 \mathrm{BrU}$ decomposition curve ( 5 to $30 \mathrm{~min}$ ).

The expected reactions for the decomposition pattern of these NBs with photon energy $(h v)$ referring to the $532 \mathrm{~nm}$ photons are presented in Table 4.

\section{Conclusions}

This work probes the response of the RNA base uracil and two of its halogenated analogues (5BrU and $5 \mathrm{FU})$ to the excitation of local gold nanoparticles (AuNPs) by $532 \mathrm{~nm}$ laser irradiation. Our results clearly show that the presence of AuNPs dramatically increases the levels of damage to the aromatic ring of modified nucleobases, as recognised via $\pi-\pi^{*}$ band suppression in UV-Vis absorption spectra recorded post-irradiation. Similarly, efficient $\pi-\pi^{*}$ band suppression was observed for all three NBs, which might indicate that the ring damage is mainly driven by processes with low selectivity such as thermal decomposition in the high temperatures surrounding of the irradiated AuNPs. Minor differences in the reaction rates of the NBs may perhaps be linked to different adsorption of the NBs to the AuNP surface affecting the reaction kinetics under pulsed laser illumination [30]. Furthermore, the laser interactions with AuNPs led to dehalogenation reactions of $5 \mathrm{BrU}$, as indicated by uracil detection in the irradiated solution. Any equivalent reactions in the irradiated $5 \mathrm{FU}$ solutions with AuNPs were too weak to detect. This observation may be attributable the different stability of the carbon-halogen bonds in an aqueous environment [34]. Alternatively, it might be linked to the molecules' different dissociative pathways induced by interactions with low-energy electrons (a major product of the irradiated AuNPs); indeed electron-attachment induced carbon-halogen bond cleavage has only previously been reported from $5 \mathrm{BrU}$ [5]. Such differences in the reactivities of $5 \mathrm{FU}$ and $5 \mathrm{BrU}$ may modify their radiosensitizing actions in the presence of irradiated AuNPs. While further work is required to fully understand the underlying mechanisms, the new observations in this work can inform future developments of combined chemo-photothermal cancer therapies exploiting AuNPs as sensitisers.

The authors acknowledge the financial support from the Fundação para a Ciência e a Tecnologia (FCT-MCTES), Radiation Biology and Biophysics Doctoral Training Programme (RaBBiT, PD/00193/2012), Applied Molecular Biosciences Unit - UCIBIO (UIDB/04378/2020) and CEFITEC 

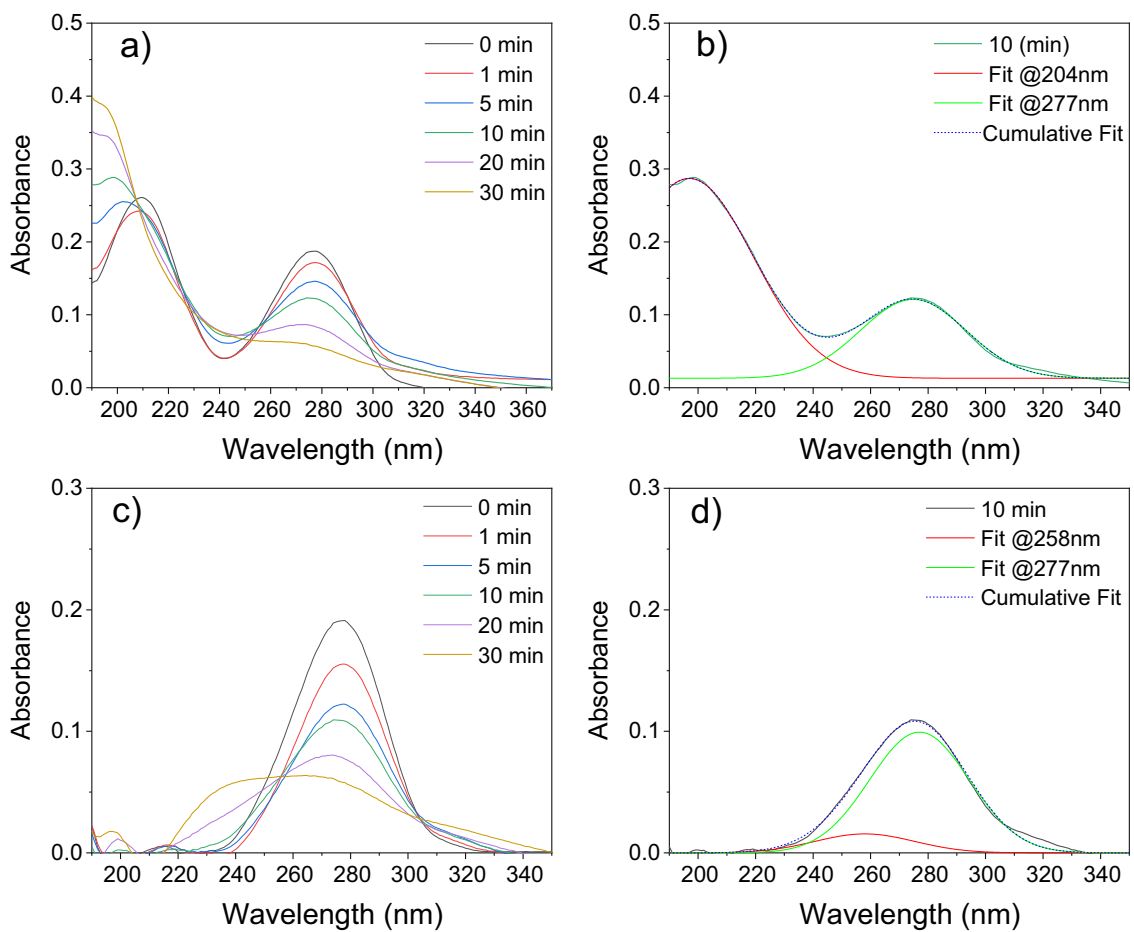

Fig. 8. Example of the data analysis: (a) corrected 5BrU+AuNPs spectra (Fig. 43c); (b) Gaussian fit of the two peaks, $\sim 204$ nm and $277 \mathrm{~nm}$, in the 10 min spectra; (c) subtraction of the band @204 nm and resulting spectra for all the irradiation periods; and (d) Gaussian fit for U and 5BrU bands @258 and $277 \mathrm{~nm}$, respectively $(Z=1 \mathrm{~mm})$.
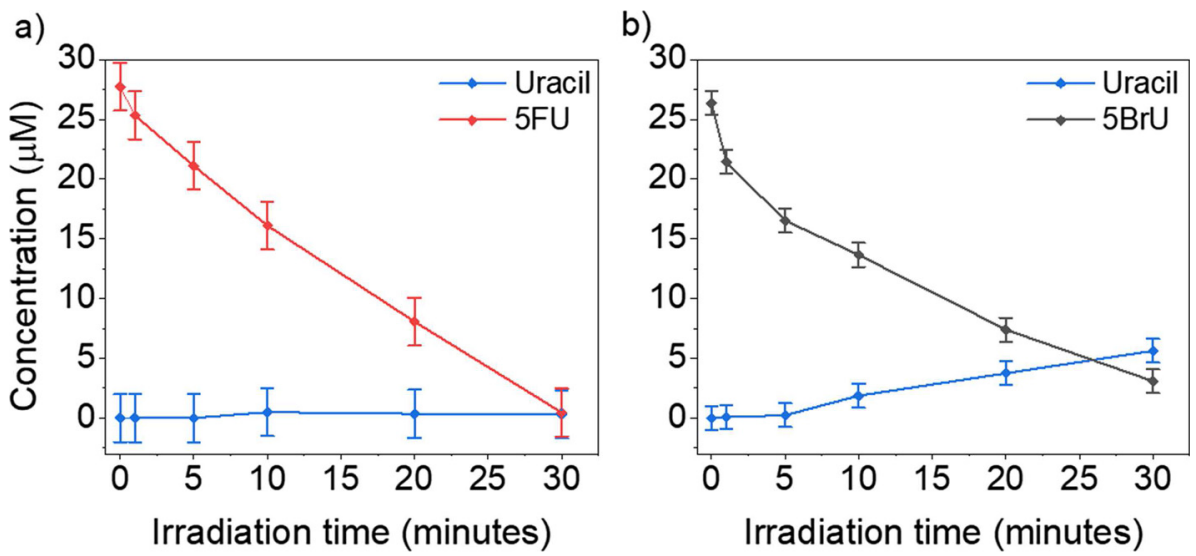

Fig. 9. Variations in the concentration of the (a) U and $5 F U$ characteristic bands after the irradiation of $5 F U$ solutions with AuNPs, and (b) U and $5 \mathrm{BrU}$ characteristic bands after the irradiation of 5FU solutions with AuNPs. The measurements were performed at laser fluence $25.5 \times 10^{13} \mathrm{~W} / \mathrm{m}^{2}(Z=1 \mathrm{~mm})$.

Table 4. Equations of the decomposition reactions expected to occur for $5 \mathrm{FU}$ and $5 \mathrm{BrU}$ in the presence of AuNPs, with the increasing of the irradiation time at $532 \mathrm{~nm}$.

\begin{tabular}{ll}
\hline Molecules & Possible reactions \\
\hline AuNPs & AuNPs $($ large $)+h v \rightarrow$ AuNPs $($ small $)+$ heat $+e^{-}$ \\
$5 \mathrm{FU}$ & Dehalogenation is inhibited or very poor \\
& $U+A u N P+h v \rightarrow$ ringfragmentation \\
$5 \mathrm{BrU}$ & $5 B r U+A u N P+h v \rightarrow B r+U$ \\
& $5 B r U+U+A u N P+h v \rightarrow$ ringfragmentation \\
& $5 B r U+h e a t \rightarrow$ ringfragmentation \\
& $5 B r U+e^{-} \rightarrow B r^{-}+U$ \\
\hline
\end{tabular}


Unit (UIDB/00068/2020), and scholarship grant number SFRH/BD/106032/2015 to Telma S. Marques. We are also grateful for the OU's logistical support and for the expert technical support provided by C. Hall, R. Seaton, T. Webley and their colleagues in building and maintaining the experiment. Finally, our thanks go to Michael Batham (OU) and Oddur Ingolffson (University of Iceland) for providing the UV-Vis spectrometer and the pulsed YAG laser, respectively.

\section{Author contribution statement}

T.S. Marques, S. Eden and N.J. Mason performed the experiments and analysed the data. R. Schürmann and I. Bald designed the experimental set-up, methodology and gave training to T.S. Marques. T.S. Marques, M.A. Śmiałek and M. Raposo wrote the first draft of the manuscript. All authors contributed to the final version of the manuscript.

Publisher's Note The EPJ Publishers remain neutral with regard to jurisdictional claims in published maps and institutional affiliations.

Open Access This is an open access article distributed under the terms of the Creative Commons Attribution License (https://creativecommons.org/licenses/by/4.0/), which permits unrestricted use, distribution, and reproduction in any medium, provided the original work is properly cited.

\section{References}

1. S. Grellet, K. Tzelepi, M. Roskamp, P. Williams, A. Sharif, R. Slade-Carter, P. Goldie, N. Whilde, M.A. Smialek, N.J. Mason, J.P. Golding, PLoS One 12, e0181103 (2017)

2. K. Haume, S. Rosa, S. Grellet, M.A. Śmiałek, K.T. Butterworth, A.V. Solov'yov, K.M. Prise, J. Golding, N.J. Mason, Cancer Nanotechnol. 7, A8 (2016)

3. R.B. Diasio, B.E. Harris, Clin. Pharmacokinet. 16, 215 (1989)

4. T.A. Rich, R.C. Shepard, S.T. Mosley, J. Clin. Oncol. 22, 2214 (2004)

5. J. Poštulka, P. Slavíček, J. Fedor, M. Fárník, J. Kočišek, J. Phys. Chem. B 121, 8965 (2017)

6. J. Rak, L. Chomicz, J. Wiczk, K. Westphal, M. Zdrowowicz, P. Wityk, M. Zyndul, S. Makurat, Ł. Golon, J. Phys. Chem. B 119, 8227 (2015)

7. S. Lehnert, Radiosensitizers and Radiochemotherapy in the Treatment of Cancer (CRC Press, Boca Raton, 2014)

8. E. Itälä, D.T. Ha, K. Kooser, E. Rachlew, M.A. Huels, E. Kukk, J. Chem. Phys. 133, (2010)

9. R. Schürmann, I. Bald, Nanoscale 9, 1951 (2017)
10. R.M. Schürmann, Doctoral thesis, Universität Potsdam, 2017

11. R.A.A.Z. Ibrahim, F.S.A. Suhail, H.K. Al-Hakeim, Nano Biomed. Eng. 10, 224 (2018)

12. M.B. Ferruz, V. Ivošev, K. Haume, L. Ellis-Gibbings, A. Traore, V. Thakare, S. Rosa, P. de Vera, V.L. Tran, A. Mika, D. Boscolo, S. Grellet, A. Verkhovtsev, B.A. Huber, K.T. Butterworth, K.M. Prise, F.J. Currell, N.J. Mason, J. Golding, E. Scifoni, G. García, F. Boschetti, F. Lux, O. Tillement, C. Louis, K. Stokbro, A.V. Solov'yov, S. Lacombe, in Nanoscale Insights into Ion-Beam Cancer Therapy, edited by A. Solov'yov (Springer, International Publishing, 2016), pp. 379-434

13. K. Haume, P. de Vera, A. Verkhovtsev, E. Surdutovich, N.J. Mason, A.V. Solov'yov, Eur. Phys. J. D 72, A116 (2018)

14. K. Haume, N.J. Mason, A.V. Solov'yov, Eur. Phys. J. D 70, A181 (2016)

15. A.V. Verkhovtsev, A.V. Korol, A.V. Solov'Yov, Phys. Rev. Lett. 114, 1 (2015)

16. A.V. Verkhovtsev, A.V. Korol, A.V. Solov'yov, J. Phys. Chem. C 119, 11000 (2015)

17. K.T. Butterworth, S.J. McMahon, F.J. Currell, K.M. Prise, Nanoscale 4, 4830 (2012)

18. L. Sanche, Radiat. Phys. Chem. 128, 36 (2016)

19. Y. Zheng, L. Sanche, Radiat. Res. 172, 114 (2009)

20. W.P. Baugh, W.D. Kucaba, Dermatologic. Surg. 31, 1290 (2006)

21. M.E. Khosroshahi, Z. Hassannejad, M. Firouzi, A.R. Arshi, Lasers Med. Sci. 30, 1913 (2015)

22. X. Liu, G. Shan, J. Yu, W. Yang, Z. Ren, X. Wang, X. Xie, H.J. Chen, X Chen, AIP Adv. 7, 025308 (2017)

23. J. Nam, S. Son, L.J. Ochyl, R. Kuai, A. Schwendeman, J.J. Moon, Nat. Commun. 9, 1 (2018)

24. L.A. Dykman, N.G. Khlebtsov, Biomed. Opt. Exp. 10, 3152 (2019)

25. D.S. Salem, M.A. Sliem, M. El-Sesy, S.A. Shouman, Y. Badr, J. Photochem. Photobiol. B Biol. 182, 92 (2018)

26. X. Huang, M.A. El-Sayed, J. Adv. Res. 1, 13 (2010)

27. G. González-Rubio, A. Guerrero-Martínez, L.M. Liz-Marzán, Acc. Chem. Res. 49, 678 (2016)

28. R. Schürmann, I. Bald, J. Phys. Chem. C 120, 3001 (2016)

29. R.L. Lundblad, F.M. Macdonald, Handbook of Biochemistry and Molecular Biology (CRC Press, 2010)

30. R. Schürmann, I. Bald, Phys. Chem. Chem. Phys. 19, 10796 (2017)

31. T.S. Marques, R. Schürmann, K. Ebel, C. Heck, M.A. Śmiałek, S. Eden, N. Mason, I. Bald, J. Chem. Phys. 152, $124712(2020)$

32. D.B. Dunn, J.D. Smith, Biochem. J. 67, 494 (1957)

33. A. Pyatenko, M. Yamaguchi, M. Suzuki, J. Phys. Chem. C 113, 9078 (2009)

34. S.W.L. Hogan, T. van Mourik, J. Comput. Chem. 38, 933 (2017) 